\title{
New Protein Clustering of Breast Cancer Tissue Proteomics Using Actin Content as a Cellularity Indicator
}

\author{
Ida Pucci-Minafra, ${ }^{,+,+\ddagger}$ Patrizia Cancemi, ${ }^{\dagger}$ Nadia Ninfa Albanese, ${ }^{\dagger}$ Gianluca Di Cara, ${ }^{\dagger}$ \\ Maria Rita Marabeti, ${ }^{\dagger}$ Antonio Marrazzo, ${ }^{\dagger, \$}$ and Salvatore Minafra ${ }^{\dagger, \neq}$ \\ Dipartimento di Oncologia Sperimentale e Applicazioni Cliniche (DOSAC), Università di Palermo, \\ Patermo, Italy, Centro di Oncobiologia Sperimentale (COBS), Palermo, Italy, and Ospedale "La Maddalena" \\ D.O. III livello, Palermo, Italy
}

Received November 15, 2007

\begin{abstract}
In the present study, we report the comparative proteome profiles of proteins solubilized from 37 breast cancer surgical tissues, normalized for the actin content. Blood-derived proteins were excluded from the analysis. Among the tumor-derived protein spots, a large proportion (39\%) was found present in all patients. These included several glycolytic enzymes, detox and heat shock proteins, members of annexin and S100 protein families, cathepsin D, and two "rare" proteins, DDAH2 involved in the angiogenesis control, and the oncogene PARK7. Other proteins, such as psoriasin, galectin1, cofilin, peroredoxins, SH3L1, and others, showed sporadic presence and high expression level, which suggests their possible role for patient stratification.
\end{abstract}

Keywords: proteomic $\bullet$ two-dimensional electrophoresis $\bullet$ peptide mass fingerprint $\bullet$ breast cancer $\bullet$ surgical tissues • protein cluster • cellularity indicator

\section{Introduction}

The exponentially increasing volume of information extracted from genomic and proteomic applications on cancer, while providing new insights into molecular composition of cancer cells and tissues, imposes new challenges on data rationalization as a tool for clinically relevant biomarker discovery. In recent years, the major agencies for cancer research have committed extensive resources to identify and make available in clinical practice biomarkers that can help to demonstrate the presence of a tumor and its malignant potential or to predict tumor behavior, prognosis, or response to treatments. ${ }^{1}$ The increasing number of detected proteins in the different laboratories conducting proteomic research has generated many candidate biomarkers with potential clinical value. However, translation from bench to bedside of the patient has proven to be more difficult than previously expected. A major reason is the complexity of the cancer as a biological system under its different hierarchical levels: genes, proteins, cells, and tissues.

Breast cancer represents the most frequent and potentially aggressive type of cancer and the leading cause of cancer deaths in women in the Western countries. Moreover, breast cancer is one of the most enigmatic and poorly predictable cancers in its evolution, likely because it includes several different forms

* To whom correspondence should be addressed. Prof. Ida Pucci-Minafra, Dipartimento di Oncologia Sperimentale e Applicazioni Cliniche; Via San Lorenzo Colli 312, 90146 Palermo, Italy. E-mail: idapucci@unipa.it. Tel/Fax: 39916806420.

† Dipartimento di Oncologia Sperimentale e Applicazioni Cliniche (DOSAC), Università di Palermo.

${ }^{\ddagger}$ Centro di Oncobiologia Sperimentale (COBS).

${ }^{\S}$ Ospedale "La Maddalena” D.O. III livello. that behave differently among patients. This biological heterogeneity is consistent with observed varied responses to therapies across patient populations.

Current clinical parameters for breast cancer diagnosis and cure are tumor size, axillary lymph node status, histological grading, and presence or absence of metastases. ${ }^{2}$ Prognostic/ predictive properties, such as estrogen and progesterone receptor status, and human epidermal growth factor receptor (HER$2 /$ neu) status, are currently used for therapeutic decision. ${ }^{3,4}$

Conversely, it is now emerging that the number of genetic mutations ${ }^{5}$ and epigenetic deregulations ${ }^{6}$ in cancer is far more higher than previously thought.

Therefore, proteomic screening for differential protein expression in subsets of tumor samples is an essential tool to generate databases, to contribute to the knowledge of biological pathways in a given cancer tissue, and to allow a molecular classification of cancer for patient stratification and biomarker discovery.

Eligibility of clinically relevant biomarkers for therapeutic use implies at least two conditions: easy accessibility from the bloodstream or tissues, and elevated expression levels with respect to the normal tissues.

In our laboratories, we have recently developed adequate extraction procedures for proteomic profiling of breast cancer tissues derived from surgery. ${ }^{7}$ A limitation of this kind of approach is the mentioned heterogeneity of breast cancer tissues, for which proteomic profiling may be influenced by extension and amount of neoplastic cells within the host stroma. To minimize this limitation and to introduce a objective criterion to compare proteomes of different tissues, the expression levels of individual proteins were normalized for actin content in each tissue extract. 

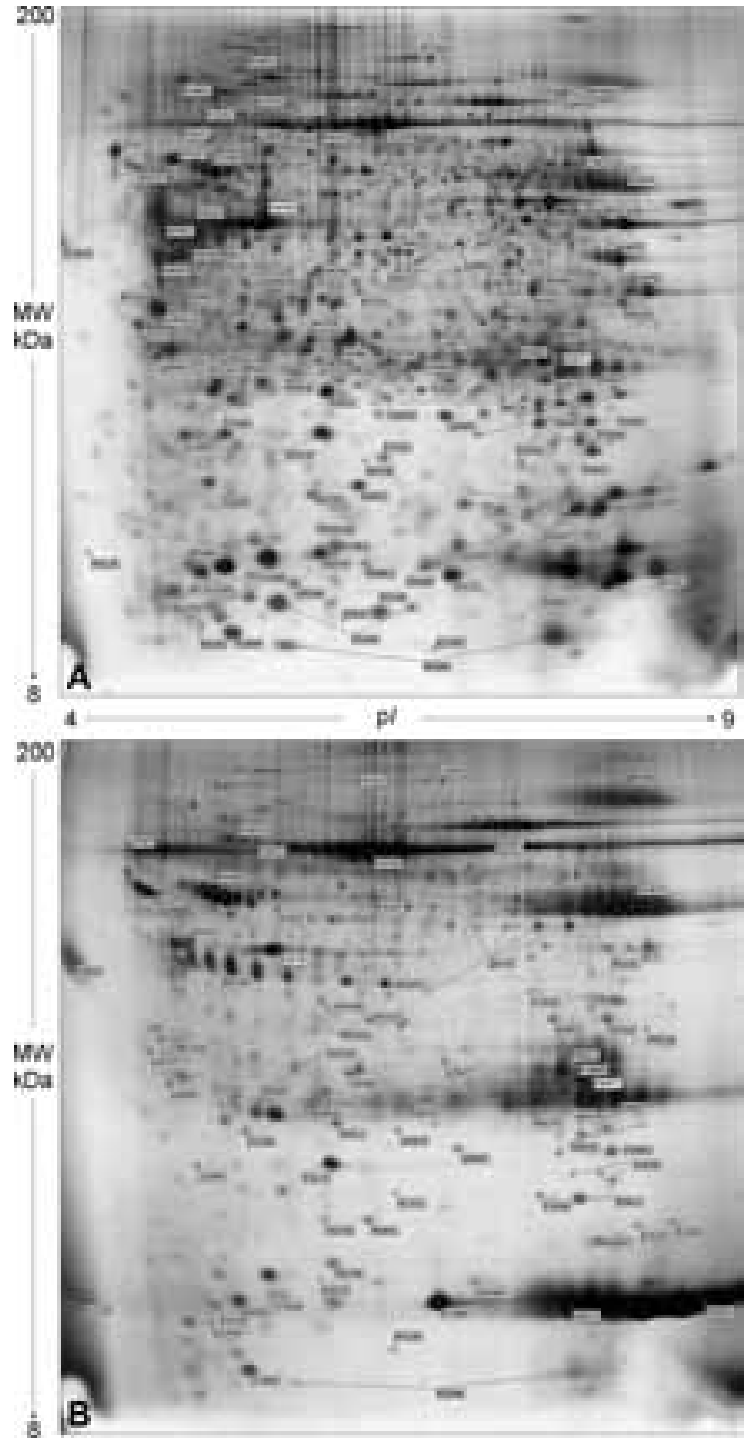

Figure 1. Representative proteomic maps of one breast cancer surgical tissue $(A)$ and its matched nontumoral adjacent tissue (B), derived from a random select patient. 2-DE separation was performed on IPG gel strips ( $18 \mathrm{~cm}, 3.5-10 \mathrm{NL}$ ) followed by the SDS-Page on a vertical linear-gradient slab gel (9-16\%T). Protein spots of known identity are labeled with the access number of the Swiss-Prot/TrEMBL database. When present, different isoforms of the same protein are jointly labeled.

In the present study, we analyzed the proteomic profiles of surgical tissues of 37 patients diagnosed for ductal infiltrating breast cancer.

Collectively, we produced a catalogue of 160 proteins. This number includes different isoforms of the same protein, as well as proteins derived from blood. The latter were filtered away before generating protein clusters according to an arbitrary scale of expression levels calculated as relative aboundance $(\% \mathrm{Vol})$ of each protein spot normalized for actin content. By this criterion, we produced six protein clusters, namely, very highly expressed, highly expressed, medium/highly expressed, medium/lowly expressed, lowly expressed, and very lowly expressed proteins.

Interestingly, a large proportion of the tumor-derived proteins $(39 \%)$ was found present in all patients. These included several glycolytic enzymes, detox and heat shock proteins,
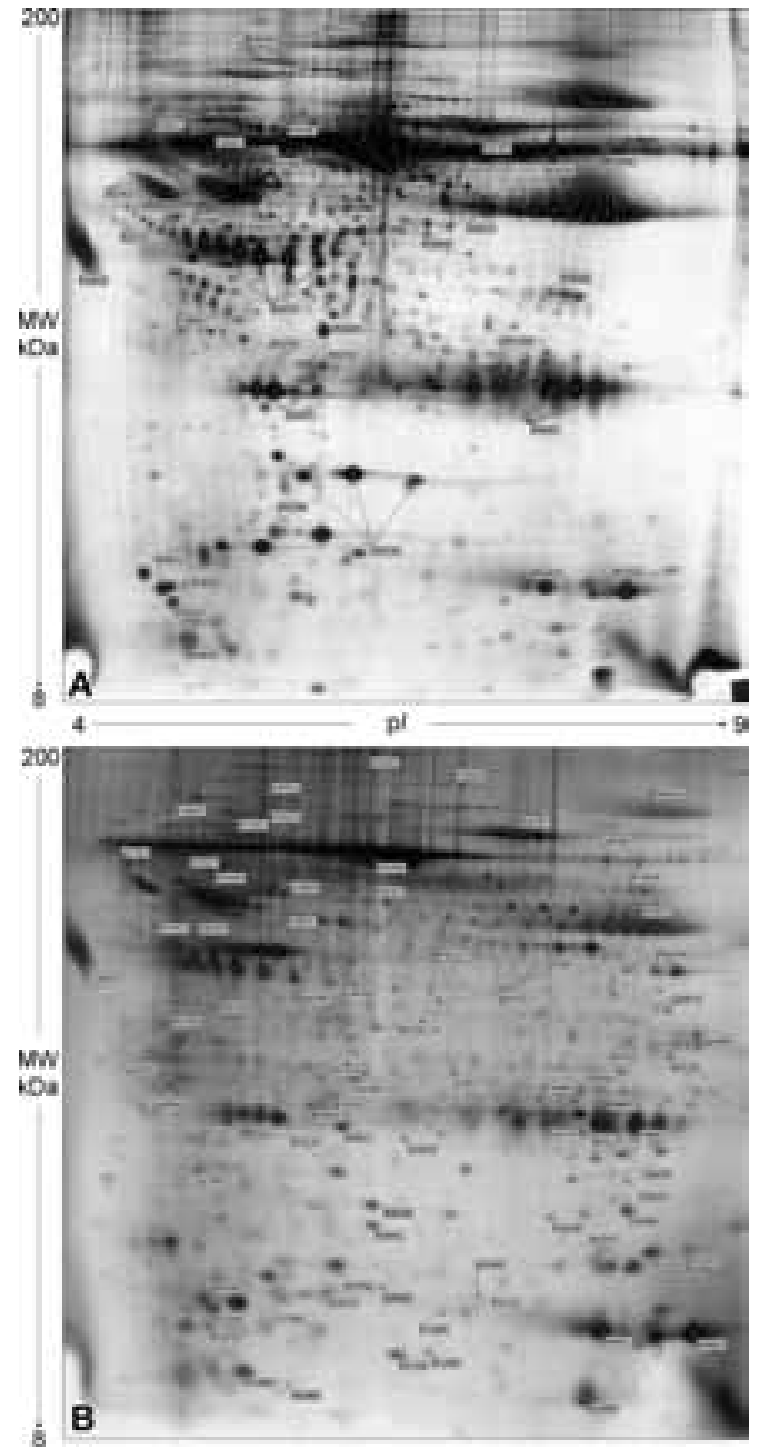

Figure 2. Proteomic maps of serum sample from a healthy subject $(A)$ and a protein extract from a breast fibroadenoma (B), showing the pattern of prominent serum proteins.

members of annexin and S100 protein families, cathepsin D, and two proteins not described before in breast cancer tissue proteomics, namely, DDAH2, involved in the angiogenesis control, and the oncogene PARK7. Other proteins, including psoriasin, galectin1, cofilin, peroredoxins, and SH3L1, showed sporadic presence and high expression level, which suggests their possible role for patient stratification.

We believe that this information may substantially contribute to the progress of protein profiling of breast cancer and help to organize subclasses for clinical applications. The statistical correlations with clinical parameters were not included in this study, because a larger population of patients is needed to generalize the results. Therefore, a contribution of efforts in this direction from several laboratories is to be encouraged.

\section{Materials and Methods}

Clinical Specimens. Sample management was performed according to the bioethical recommendations. Aliquots of breast cancer and its adjacent nontumoral tissues were obtained during surgical intervention and immediately frozen in 

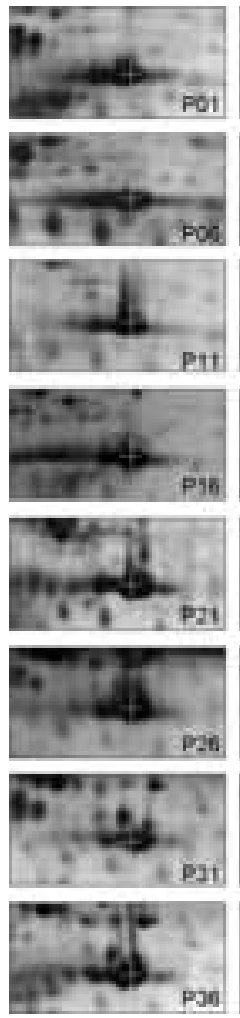
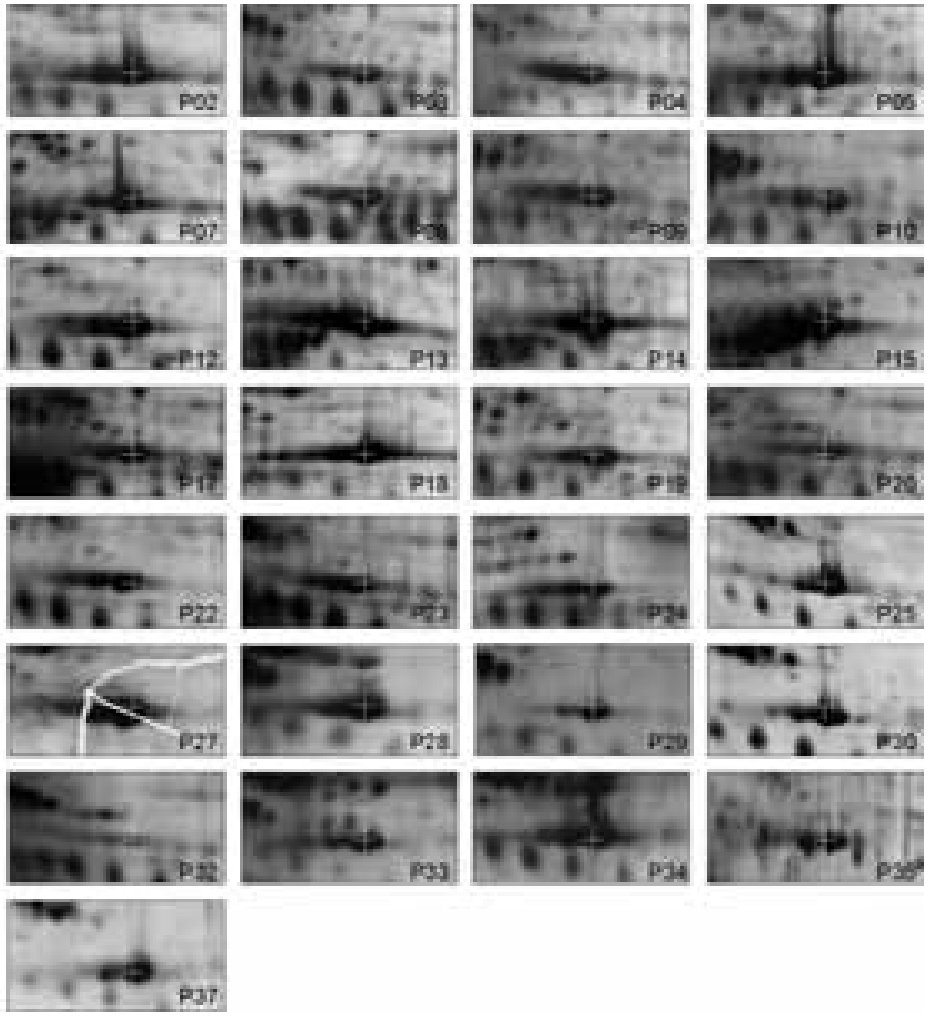

Figure 3. Panel showing an experimental window from ImageMaster 2D Platinum software containing the sections of actin spots (white crosses) from the matched two-dimensional gels of the 37 breast cancer surgical tissues, indicated from P01 to P37.

liquid nitrogen and stored at $-80{ }^{\circ} \mathrm{C}$ until use. The patients did not receive any cytotoxic/endocrine treatment prior to surgery. In each cases, nontumoral tissue was located at least $5 \mathrm{~cm}$ away from the primary tumor. Diagnosis of ductal breast cancer (G2/G3) was confirmed histopathologically.

Sample Preparations. The frozen breast tissue samples were washed several times with phosphate-buffered saline and homogenized in RIPA buffer (50 mM Tris, pH 7.5, 0.1\% Nonidet P-40, 0.1\% deoxycholate, $150 \mathrm{mM} \mathrm{NaCl}$, and $4 \mathrm{mM}$ EDTA) and a mixture of protease inhibitors $(0.01 \%$ aprotinin, $10 \mathrm{mM}$ sodium pyrophosphate, $2 \mathrm{mM}$ sodium orthovanadate, and 1 mM PMSF). The extraction was carried out overnight at $4{ }^{\circ} \mathrm{C}$ with the same buffer. The total cellular lysate was centrifuged at $15000 \mathrm{rpm}$ for $20 \mathrm{~min}$ to clear debris, and the supernatant was dialysed against ultrapure distilled water, lyophilised, and stored at $-80{ }^{\circ} \mathrm{C}$ until analysis. Protein concentration in the cellular extracts was determined using the Bradford method. ${ }^{8}$

Two-Dimensional Gel Electrophoresis. The proteins extracted from breast cancer tissue and normal adjacent tissue were solubilized in a buffer containing 4\% CHAPS, $40 \mathrm{mM}$ Tris, and $65 \mathrm{mM}$ DTE in $8 \mathrm{M}$ urea. Aliquots of $45 \mu \mathrm{g}$ (analytical gels) or $1.5 \mathrm{mg}$ (preparative gels) of total proteins were separately mixed with $350 \mu \mathrm{L}$ of rehydration solution containing $8 \mathrm{M}$ urea, $2 \%$ CHAPS, 10 mM DTE, and 0.5\% carrier anpholytes (Resolyte 3.5-10) and applied for IEF using commercial sigmoidal IPG strips, $18 \mathrm{~cm}$ long with $\mathrm{pH}$ range 3.5-10. The second dimension was carried out on $9-16 \%$ linear gradient polyacrylamide gels (SDS-PAGE), and the separated proteins were visualized by ammoniacal silver staining. Two or three replicas were performed for each patient.

Image Acquisition and Data Analysis. Silver-stained gels were digitized using a computing densitometer and analyzed with ImageMaster 2D Platinum software (Amersham Bio- sciences, Sweden). Gel calibration was carried out using an internal standard and the support of the ExPaSy molecular biology server, as described. ${ }^{9}$ Quantitative variations in protein expression levels were calculated as the volume of the spots (i.e., integration of optical density over the spot area). To correct for differences in gel staining, spot volumes relative to the sum of the volume of all spots on each gel $(\% \mathrm{Vol})$ were calculated by the software. Furthermore, for comparative profiling, individual spot \%Vol was normalized for the actin \%Vol value. The resulting figure was considered an indicator of protein expression level and utilized for protein clusterization.

N-terminal Protein Sequencing. N-terminal microsequencing was performed by automated Edman degradation in a Protein Sequencer (Procise 491, Applied Biosystems) on protein samples electrotransferred onto PVDF membranes at $50 \mathrm{~V}$ for $30 \mathrm{~min}$ at $4{ }^{\circ} \mathrm{C} .{ }^{10}$ The Swiss-Prot/TrEMBL/BLAST databases were used for online sequence similarity search at http://www. expasy.ch/tools/\#similarity.

Matrix-Assisted Laser Desorption Ionization-Time of Flight (MALDI-TOF). Mass spectrometric sequencing was carried out after in-gel digestion of protein spots, using sequencing-grade trypsin $(20 \mu \mathrm{g} / \mathrm{vial})$, according to the method of Shevchenko et al. ${ }^{11}$ with some modification. ${ }^{10}$ The tryptic peptide extracts were dried in a vacuum centrifuge and redissolved in $10 \mu \mathrm{L}$ of $0.1 \%$ trifluoroacetic acid (TFA). The matrix, $\alpha$-cyano-4-hydroxycinnamic acid (HCCA), was purchased from Sigma-Aldrich. A saturated solution of HCCA (1 $\mu \mathrm{L})$ at $2 \mathrm{mg} / 200 \mu \mathrm{L}$ in $\mathrm{CH}_{3} \mathrm{CN} / \mathrm{H}_{2} \mathrm{O}(50: 50(\mathrm{v} / \mathrm{v}))$ containing $0.1 \%$ TFA was mixed with $1 \mu \mathrm{L}$ of peptide solution on the MALDI plate and left to dry. MALDI-TOF mass spectra were recorded on a Voyager DE-PRO (Applied-Biosystems) mass spectrometer, in the 500-5000 Da mass range, using a minimum of 100 shots of laser per spectrum. Delayed extraction source and reflector 


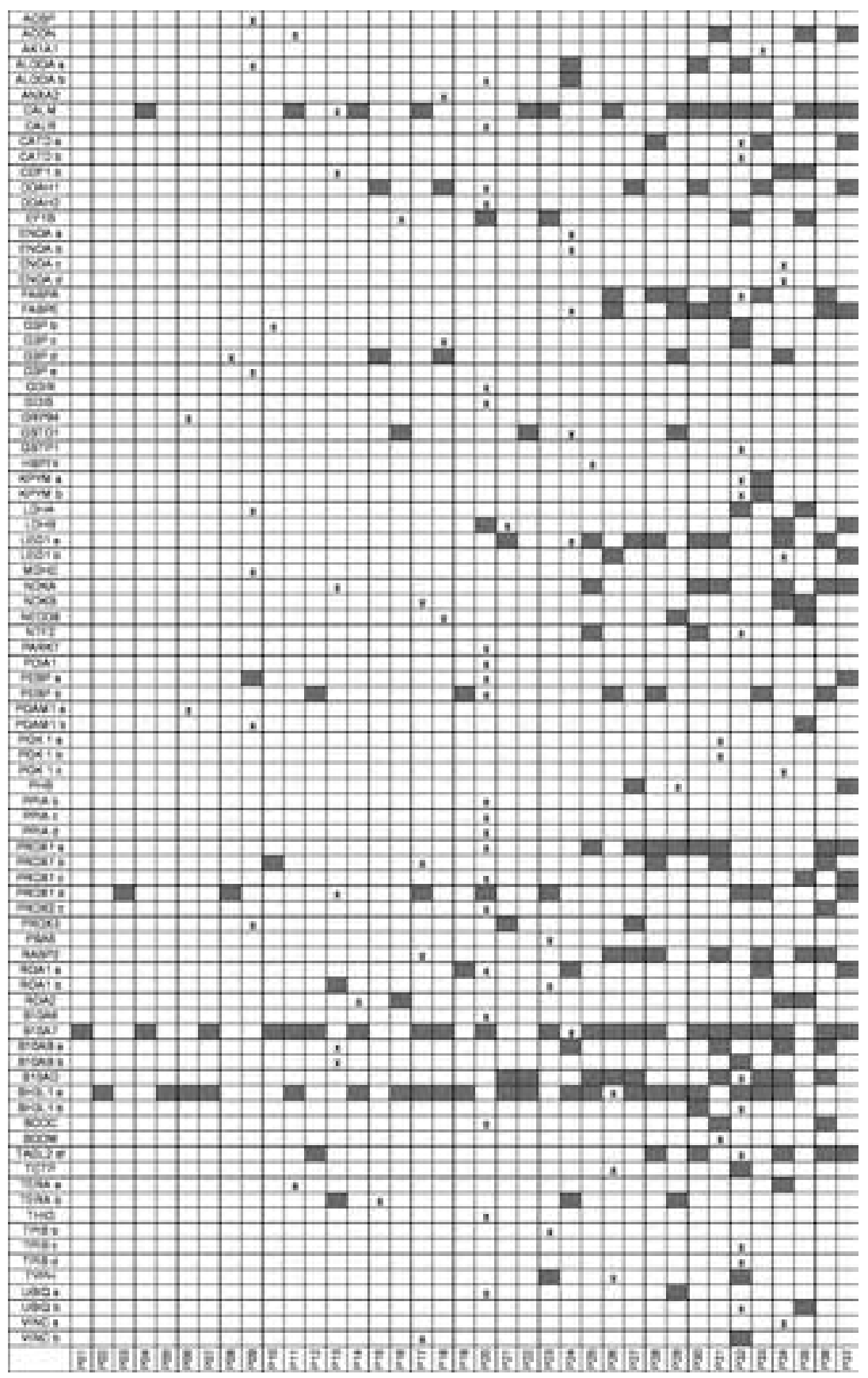

Figure 4. Diagram illustrating the distribution of the identified proteins among the 37 patients, designated from P01 to P37. Alphabetical letters following protein abbreviated names indicate the different isoforms, starting from the more acidic one. The black boxes indicate absence of proteins in the map of the corresponding patient, and the crosses in each box indicate the patient expressing the given protein at its highest level.

equipment allowed sufficient resolution to consider $\mathrm{MH}^{+}$of monoisotopic peptide masses. Internal calibration was done using trypsin autolysis fragments at $\mathrm{m} / z$ 842.5100, 1045.5642, and 2211.1046 Da. Peptide mass fingerprinting was compared to the theoretical masses from the Swiss-Prot or NCBI sequence databases using Mascot (http://www.matrixscience.com/). Typical search parameters were as follows: $\pm 50 \mathrm{ppm}$ of mass tolerance, carbamidomethylation of cysteine residues, one missed enzymatic cleavage for trypsin, a minimum of four peptide mass hits was required for a match, methionine residues could be considered in oxidized form, no restriction was placed on the isoelectric point of the protein, and a protein mass range from 5 to $100 \mathrm{kDa}$ was allowed.
Western Blotting. For immune detection, the gels (9-16\% linear gradient SDS-PAGE) were electrotransferred onto nitrocellulose membrane (HyBond ECL, Amersham) at $50 \mathrm{~V}$ for $1 \mathrm{~h}$ at $4{ }^{\circ} \mathrm{C}$. After electrotransfer, the membranes were blocked with $5 \%$ of dry milk in T-TBS for $1 \mathrm{~h}$ and probed with one of the following antibodies (Santa Cruz): monoclonal anti-PARK7, anti-S10A7, anti-GDIR, and goat polyclonal anti-DDAH1 and anti-DDAH2, at 1:1000 diluted with $1 \%$ milk in T-TBS. Following incubation with anti-mouse or anti-goat peroxidase-linked antibody (Amersham), the reaction was revealed using enhanced chemioluminiscence (Pierce), according the manufacturer's instructions. 


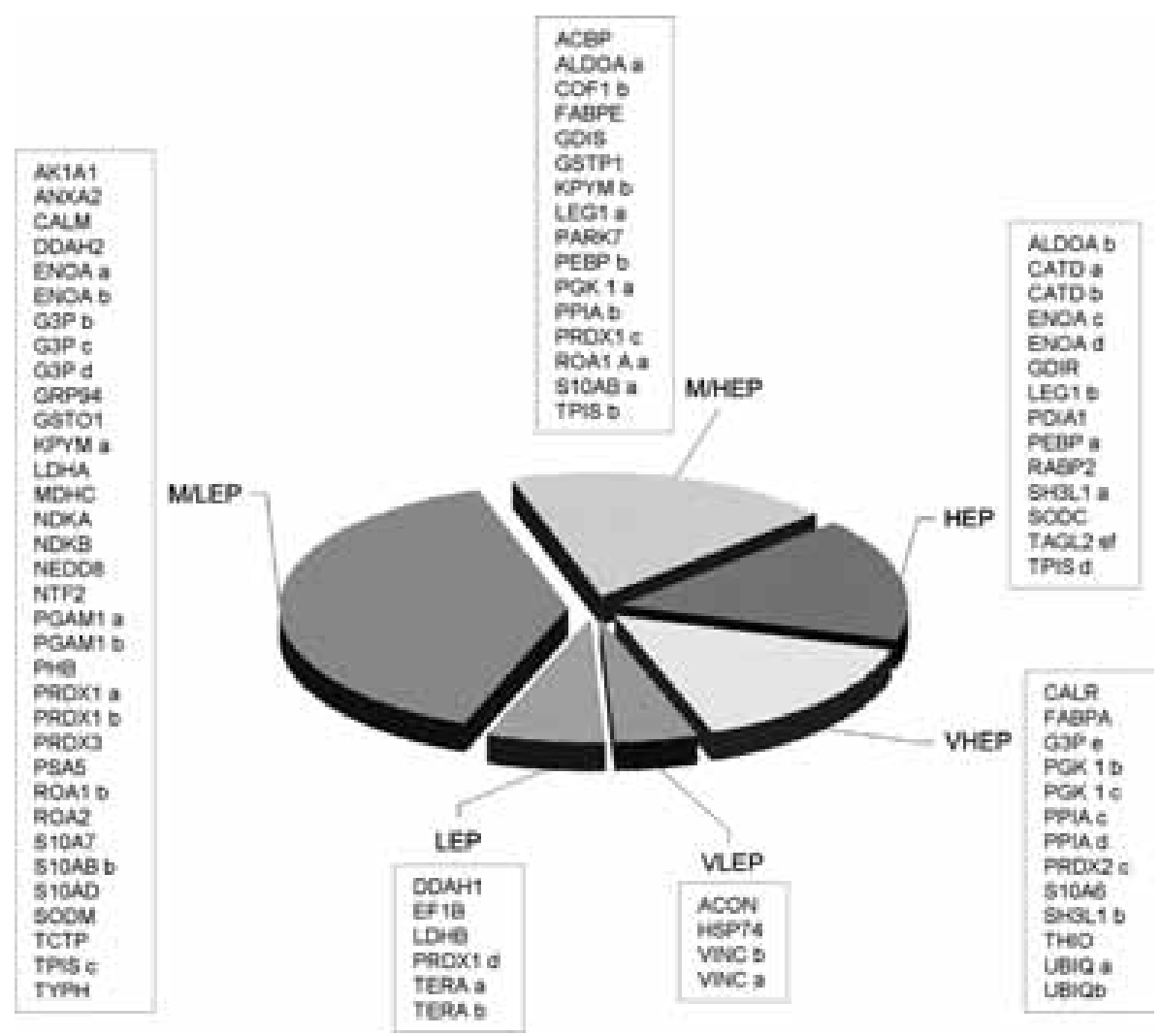

Figure 5. Protein clusters were generated by sorting proteins with the highest expression level among the 37 patients. The protein spot expression level was calculated as the \%Vol normalized for actin content, of each patient map, after subtraction of blood-derived proteins. The range of values are expressed on an arbitrary scale, from 0.05 to $>1$, and indicated as: VHEP, very highly expressed proteins (range 0.8-1); HEP, highly expressed proteins (0.6-0.79); M/HEP, medium/highly expressed proteins (0.4-0.59); M/LEP, medium/ lowly expressed (0.2-0.39); LEP, lowly expressed (0.1-0.19); VLEP, very lowly expressed (0.05-0.09).

\section{Results and Discussion}

The methodology described to isolate proteins from the surgical specimens allows the releasing of salt-soluble proteins from cellular and extracellular compartments. No efforts were made to extract ECM components, because they not pertinent to the present research.

We have recently reported the comparative proteomic profiles of 13 pairs of surgical samples obtained from patients with ductal infiltrating breast cancer. ${ }^{7}$ As expected, the contribution of cellular proteins to the proteomic complexity, was much higher in the tumor extracts than in their paired nontumoral conterparts. As an example, panels A and B of Figure 1 show two proteomic maps, respectively, generated from a breast cancer tissue extract and its paired nonaffected tissue. The labels on the map indicate the access number (Swiss-Prot/TrEMBL database) of the identified proteins. A remarkable difference between the two proteomic maps for protein spot composition is clearly observed. We suggest that this different pattern is due to the reduced amount of parenchima in the mammary gland of healthy adult women, opposed to the abundant cell population present in the tumor core. Indeed, the proteomic profile of nontumoral tissue extracts reveals the almost exclusive presence of serum and blood proteins, consisting mainly of albumin, immunoglubulins, and other globulins. Figure 2A shows the serum proteome of a healthy subject compared with the normal tissue extract. Similarly, the fibroadenoma proteome, a benign breast lesion, dispalys a very small amount of cellular proteins with respect to blood-derived proteins (Figure 2B).
The collection of proteins so far identified in our tumor samples contains 160 proteins, including isoelectric variants and bood-derived proteins (supplemental Table 1 in Supporting Information). When present, the different isoforms of the same protein are indicated by alphabetical letters, starting from the more acidic one.

Since the cellularity of the tumor biopsy, that is, cell densities within an area of the surgical sample, may be very variable among the different subjects, measurements of relative expression levels (\%Vol) of individual protein spots were normalized in each map for actin content.

Figure 3 shows an experimental window containing the sections of actin spots from the 37 two-dimensional gels, testifying the variability of actin amount among the proteomic maps of our study, likely as an effect of different degree of cellularity in the samples examined, under the same quantity of protein loading in the 2D-IPG.

Protein Presence-Absence. As shown in Figure 1A, the tumor samples, besides the proteins of cellular origin, contain variable amount of blood-derived proteins. The latter were filtered out from our catalogue. The remaining amount, likely representing tumor-related proteins, accounted for 87, 34 of which, that is $39 \%$, were expressed in all samples. Figure 4 is a diagram illustrating the distribution of the identified proteins among the 37 patients, indicated from P01 to P37. The black boxes indicate absence of proteins in the map of the corresponding patient, and the crosses in each box indicate the patient expressing the given protein at its highest level among the 37 chosen ones. 


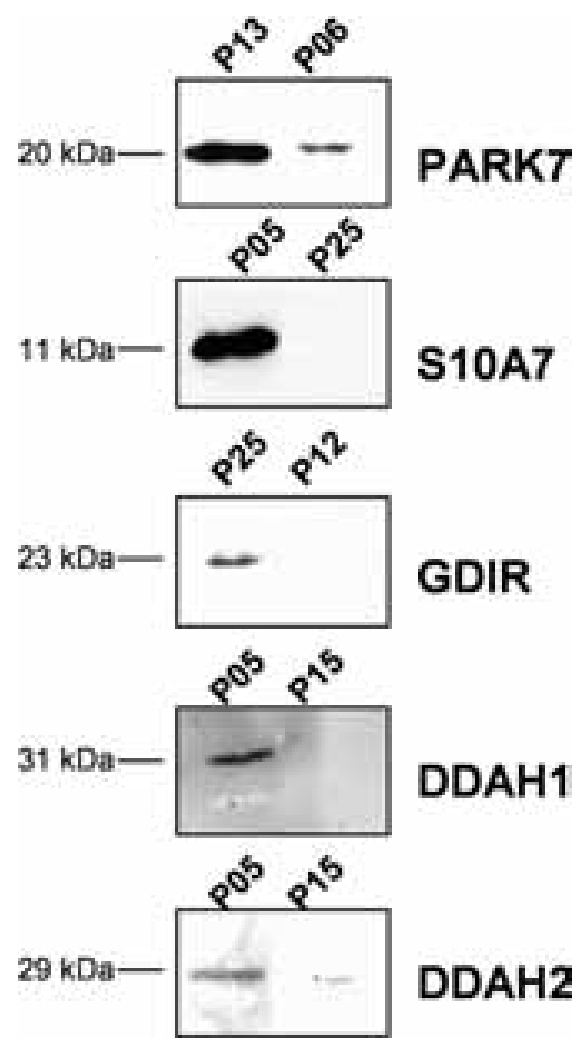

Figure 6. Panel showing a Western blot assay to validate a group of new proteins detected in the breast cancer proteomics by peptide mass fingerprint. Namely, PARK7, S10A7, GDIR, DDAH1, and DDAH2. The validation was performed on couples of patients, chosen among the ones indicated by P01-P37 in the diagram in Figure 4, respectively, showing high and low level (or absence) of the cited proteins. On the left: molecular markers.

Interestingly, and in agreement with current knowledge, ${ }^{9,12}$ a major portion of ubiquitous proteins belong to the glycolytic metabolism (12/34, i.e., 35\%, including isoforms); seven protein forms include 2 protein foldings (PDA1, and 3 isoforms of PPIA), a calcium-binding chaperone (CALR) and 2 heat shock proteins (HSP74 and GRP94); four proteins are represented by 3 members of redox-defense machinery (SOD, THIO, GSTP1), and one recently identified oncogene PARK7, a master regulator of antioxidant transcriptional responses. ${ }^{13}$ Other intersting components of this proteomic profiling are 2 regulators of the transduction machinery (GDIR and GDIS), the Dimethylargininase 2 (DDAH2), a relevant enzyme involved in the angiogenesis control, ${ }^{14}$ and a subgroup of proteins like ANXA2, S10A6 calcium-binding protein, and vinculin which individually and/ or in synergy are important players of events related to invasion and metastasis. ${ }^{15-19}$

Finally, another ubiquitously expressed protein was the cathepsin D, a controversial prognostic marker for breast cancer progression. ${ }^{20}$ This was detected under two isoelectric variants with $\mathrm{p} I$ of 5.29 and 5.69 , respectively, the latter being usually the more aboundant.

It is worth to mention that several ubiquitous proteins are multifunctional, therefore, may be involved in more metabolic pathways, besides their major role in the cell. Indeed, HSP70 protein family, GRP94, and CALR may also exert a role in the tumor immune-responses. ${ }^{21}$ Similarly, G3P, ENOA, PGAM1, and TPIS, besides their role in the glycolytic pathway, are known to exploit several diversified functions; that is, G3P2, is known to facilitate membrane fusion, microtubule bundling, and it is capable of nucleic acid binding; ${ }^{22,23}$ alpha-enolase functions as a plasminogen receptor on some cell types, ${ }^{24}$ while PGAM1 has been shown to substain indefinite proliferation in embryonic fibroblasts. ${ }^{25}$ It has been also reported that triosephosphate isomerase activity, which play a key role in the interconnection between the pentose phosphate pathway and the lipid metabolism, tends to be higher expressed in immature myeloid cells. ${ }^{26,27}$ Recently, it has also been demonstrated that calreticulin has a role in regulating p53 function by affecting its rate of degradation and nuclear localization. ${ }^{28}$

Protein Clustering According to the Expression Level. After subtraction of proteins derived from blood, and normalization for actin contents, we sorted the proteins by the highest expression level among the 37 analyzed patients. These proteins were then grouped according to an arbitrary scale, with values (\%Vol normalized for actin) ranging from 0.05 to $>1$. By this criterion, we generated six protein clusters (Figure 5): (1) very highly expressed proteins (VHEP, 14.94\%), (2) highly expressed proteins (HEP, 16.09\%), (3) medium/highly expressed proteins (M/HEP, 18.39\%), (4) medium/lowly expressed (M/LEP, 39.08\%), (5) lowly expressed (LEP, 6.89\%), and (6) very lowly expressed (VLEP, 4.59\%).

Remarkably, among the VHEP and HEP clusters are some glycolytic enzymes, proteins of cellular defenses, proteins belonging to the S100 family, and the 2 isoforms of CATD. These two groups also include proteins with a very peculiar property: their appearance is sporadic, but when present, their expression is never low. This is the case, among others, of galectin 1 (LEG1), several proteins of the S100-calcium binding family, and SH3L1, a gene product belonging to the SH3BGR (SH3 domain-binding glutamic acid-rich-like protein family) and structurally related to thioredoxin (Trx) super family. ${ }^{29}$

Psoriasin (S10A7) is one of the most variable proteins among the 37 patients. It is highly expressed in 15/37 patients, and its presence may be predictive of invasive and metastaic progression of the tumor. ${ }^{30-32}$

The clusters of M/LEP (39.08\%) and M/HEP (18.39\%) all together represent the major part of the proteins $(57.47 \%)$. It is worth to notice also the presence of "rare" proteins like TYPH/ PD-ECGF known to be angiogenic growth factors in vitro and in vivo ${ }^{33}$ and the cited PARK7.

A general observation concerns the differential expression of isoelectric variants of the same proteins: very often the level of intensity of these forms is not the same in different patients, suggesting the existence of epigenetic factors, differentially operating in the various tumor environments.

Western Blot Validation of Selected Proteins. Finally, immunological assays were performed to confirm the results obtained by peptide mass fingerprinting in relation to a set of new proteins, namely, PARK7, S10A7, GDIR, DDAH1, and DDAH2 (Figure 6). The validation was performed on couples of patients, chosen among the ones indicated by P01-P37 in the diagram in Figure 4, respectively, showing high and low level (or absence) of the cited proteins.

\section{Concluding Remarks}

A good performance of proteomic profiling and protein detection by sequencing methodologies needs remarkable efforts, both as human and economical resources. Thorough and courageous selection of results is necessary to ensure high quality and reliable data. Therefore, to achieve the goal for 
translational proteomics, that is, differential diagnosis, prognosis, prediction, and monitor course, collective efforts are to be encouraged. This study represents a contribution in this direction.

Acknowledgment. The present research is part of the project DIAMOL (Por Sicilia misura 3.4). The authors thank the numerous persons who have given their support to this work and, in particular, Dr. Pietra Taormina, Dr. Elena Roz, Dr. Carmelo Lupo, Dr. Maria Vera Lo Presti, Dr Silvana Caricato, Dr. Lara Fricano, Miss Gilda Barbera, Mr. Vito Balsamo, and all the Breast Unit Staff directed by Dr. A. Marrazzo, at the Maddalena Hospital.

Note Added after ASAP Publication. Cathepsin D was misspelled in the Abstract and Introduction on the version published ASAP on 2/20/2008. The correct version was published 3/7/2008.

Supporting Information Available: Table listing the collection of proteins so far identified in our tumor samples containing 160 proteins, including isoelectric variants and bood-derived proteins. This material is available free of charge via the Internet at http://pubs.acs.org.

\section{References}

(1) Hinestrosa, M. C.; Dickersin, K.; Klein, P.; Mayer, M.; Noss, K.; Slamon, D.; Sledge, G.; Visco, F. M. Shaping the future of biomarker research in breast cancer to ensure clinical relevance. Nat. Rev. Cancer 2007, 7 (4), 309-315.

(2) Donegan, W. L. Tumor-related prognostic factors for breast cancer. CA Cancer J. Clin. 1997, 47 (1), 28-51.

(3) Goldhirsch, A.; Wood, W. C.; Gelber, R. D.; Coates, A. S.; Thürlimann, B.; Senn, H. J. Meeting highlights: updated international expert consensus on the primary therapy of early breast cancer. J.Clin.Oncol. 2003, 21 (17), 3357-3365.

(4) Hussain, S. A.; Palmer, D. H.; Spooner, D.; Rea, D. W. Molecularly targeted therapeutics for breast cancer. BioDrugs 2007, 21 (4), 215224.

(5) Greenman, G.; Stephens, P.; Smith, R.; Gillian, L.; Dalgliesh, G. L.; et al. Patterns of somatic mutation in human cancer genomes. Nature 2007, 446 (7132), 153-158.

(6) Malik, K.; Brown, W. Epigenetic gene deregulation in cancer. Br. J. Cancer 2000, 83 (12), 1583-1588.

(7) Pucci-Minafra, I.; Cancemi, P.; Marabeti, M. R.; Albanese, N. N.; Di Cara, G.; Taormina, P.; Marrazzo, A. Proteomic profiling of 13 paired ductal infiltrating breast carcinomas and non-tumoral adjacent counterparts. Proteomics Clin. Appl. 2007, 1, 118-129.

(8) Bradford, M. M. A rapid and sensitive method for the quantitation of microgram quantities of protein utilizing the principle of protein-dye binding. Anal. Biochem. 1976, 7 (72), 248-254.

(9) Pucci-Minafra, I.; Fontana, S.; Cancemi, P.; Alaimo, G.; Minafra, S. Proteomic patterns of cultured breast cancer cells and epithelial mammary cells. Ann. N.Y. Acad. Sci. 2002, 963, 122-139.

(10) Pucci-Minafra, I.; Cancemi, P.; Fontana, S.; Minafra, L.; Feo, S.; Becchi, M.; Freyria, A. M.; Minafra, S. Expanding the protein catalogue in the proteome reference map of human breast cancer cells. Proteomics 2006, 6 (8), 2609-2625.

(11) Shevchenko, A.; Wilm, M.; Vorm, O.; Mann, M. Mass spectrometric sequencing of proteins silver-stained polyacrylamide gels. Anal. Chem. 1996, 68 (5), 850-858.

(12) Altenberg, B.; Greulich, K. O. Genes of glycolysis are ubiquitously overexpressed in 24 cancer classes. Genomics 2004, 84 (6), 10141020.

(13) Clements, C. M.; McNally, R. S.; Conti, B. J.; Mak, T. W.; Ting, J. P. DJ-1, a cancer- and Parkinson's disease-associated protein, stabilizes the antioxidant transcriptional master regulator Nrf2. Proc. Natl. Acad. Sci. U.S.A. 2006, 103 (41), 15091-15096.

(14) Hasegawa, K.; Wakino, S.; Tanaka, T.; Kimoto, M.; Tatematsu, S.; Kanda, T.; Yoshioka, K.; Homma, K.; Sugano, N.; Kurabayashi, M.; Saruta, T.; Hayashi, K. Dimethylarginine dimethylaminohydrolase 2 increases vascular endothelial growth factor expression through
Sp1 transcription factor in endothelial cells. Arterioscler. Thromb. Vasc. Biol. 2006, 26 (7), 1488-1494.

(15) Díaz, V. M.; Hurtado, M.; Thomson, T. M.; Reventos, J.; Paciucci, R. Specific interaction of tissue-type plasminogen activator (t-PA) with annexin II on the membrane of pancreatic cancer cells activates plasminogen and promotes invasion in vitro. Gut 2004, 53 (7), 993-1000.

(16) Ortiz-Zapater, E.; Peiró, S.; Roda, O.; Corominas, J. M.; Aguilar, S.; Ampurdanés, C.; Real, F. X.; Navarro, P. Tissue plasminogen activator induces pancreatic cancer cell proliferation by a noncatalytic mechanism that requires extracellular signal-regulated kinase $1 / 2$ activation through epidermal growth factor receptor and annexin A2. Am. J. Pathol. 2007, 170 (5), 1573-1584.

(17) Rehman, I.; Cross, S. S.; Azzouzi, A. R.; Catto, J. W.; Deloulme, J. C.; Larre, S.; Champigneuille, J.; Fromont, G.; Cussenot, O.; Hamdy, F. C. S100A6 (Calcyclin) is a prostate basal cell marker absent in prostate cancer and its precursors. Br. J. Cancer 2004, 91 (4), 739744.

(18) Vimalachandran, D.; Greenhalf, W.; Thompson, C.; Lüttges, J.; Prime, W.; Campbell, F.; Dodson, A.; Watson, R.; CrnogoracJurcevic, T.; Lemoine, N.; Neoptolemos, J.; Costello, E. High nuclear S100A6 (Calcyclin) is significantly associated with poor survival in pancreatic cancer patients. Cancer Res. 2005, 65 (8), 3218-3225.

(19) Liu, M.; Oberg, K.; Zhou, Y. Expression and function of vinculin in neuroendocrine tumors. Tumor Biol. 2007, 28 (4), 96-204.

(20) Zhang, Y. G.; Du, J.; Tian, X. X.; Zhong, Y. F.; Fang, W. G. Expression of E-cadherin, beta-catenin, cathepsin D, gelatinases and their inhibitors in invasive ductal breast carcinomas. Chin. Med. J. 2007, 120 (18), 1597-1605.

(21) Obeid, M.; Tesniere, A.; Ghiringhelli, F.; Fimia, G. M.; Apetoh, L.; Perfettini, J. L.; Castedo, M.; Mignot, G.; Panaretakis, T.; Casares, N.; Métivier, D.; Larochette, N.; van Endert, P.; Ciccosanti, F.; Piacentini, M.; Zitvogel, L.; Kroemer, G. Calreticulin exposure dictates the immunogenicity of cancer cell death. Nat. Med. 2007, 13 (1), 54-61.

(22) Sirover, M. A. New insights into an old protein: the functional diversity of mammalian glyceraldehyde-3-phosphate dehydrogenase. Biochim. Biophys. Acta 1999, 1432 (2), 159-184.

(23) Savagner, P. Leaving the neighborhood: molecular mechanisms involved during epithelial-mesenchymal transition. BioEssays 2001, 23 (10), 912-923.

(24) Miles, L. A.; Dahlberg, C. M.; Plescia, J.; Felez, J.; Kato, K.; Plow, E. F. Role of cell-surface lysines in plasminogen binding to cells: identification of alpha-enolase as a candidate plasminogen receptor. Biochemistry 1991, 30 (6), 1682-1691.

(25) Kondoh, H.; Lleonart, M. E.; Gil, J.; Wang, J.; Degan, P.; Peters, G.; Martinez, D.; Carnero, A.; Beach, D. Glycolytic enzymes can modulate cellular life span. Cancer Res. 2005, 65 (1), 177-185.

(26) Yin, M. Y.; Gao, X. Z.; Wang, Z. Q.; Preisler, H. D. Studies of the proliferation and differentiation of immature myeloid cells in vitro: 4: Preculture proto-oncogene expression and the behaviour of myeloid leukemia cells in vitro. Cell Biochem. Funct. 1991, 9 (1), $39-47$.

(27) Orosz, F.; Oláh, J.; Ovádi, J. Triosephosphate isomerase deficiency: facts and doubts. IUBMB Life 2006, 58 (12), 703-715.

(28) Mesaeli, N.; Phillipson, C. Impaired p53 expression, function, and nuclear localization in calreticulin-deficient cells. Mol. Biol. Cell 2004, 15 (4), 1862-1870.

(29) Xu, C.; Zheng, P.; Shen, S.; Xu, Y.; Wei, L.; Gao, H.; Wang, S.; Zhu, C.; Tang, Y.; Wu, J.; Zhang, Q.; Shi, Y. NMR structure and regulated expression in APL cell of human SH3BGRL3. FEBS Lett. 2005, 579 (13), 2788-2794.

(30) Al Haddad, S.; Zhang, Z.; Leygue, E.; Snell, L.; Huang, A.; Niu, Y.; Hiller-Hitchcock, T.; Hole, K.; Murphy, L. C.; Watson, P. H. Psoriasin (S100A7) expression and invasive breast cancer. Am. J. Pathol. 1999, 155 (6), 2057-2066.

(31) Emberley, E. D.; Murphy, L. C.; Watson, P. H. S100A7 and the progression of breast cancer. Breast Cancer Res. 2004, 6 (4), 153159.

(32) Krop, I.; März, A.; Carlsson, H.; Li, X.; Bloushtain-Qimron, N.; Hu, M.; Gelman, R.; Sabel, M. S.; Schnitt, S.; Ramaswamy, S.; Kleer, C. G.; Enerbäck, C.; Polyak, K. A putative role for psoriasin in breast tumor progression. Cancer Res. 2005, 65 (24), 11326-11334.

(33) Toi, M.; Inada, K.; Hoshina, S.; Suzuki, H.; Kondo, S.; Tominaga, T. Vascular endothelial growth factor and platelet-derived endothelial cell growth factor are frequently coexpressed in highly vascularized human breast cancer. Clin. Cancer Res. 1995, 1 (9), 961-964.

\section{PR700748M}

